\title{
СРАВНИТЕЛЬНЫЙ АНАЛИЗ СОДЕРЖАНИЯ МЕЖФАЗНЫХ ОБЛАСТЕЙ И ИХ ВЛИЯНИЕ НА СВОЙСТВА НАНОКОМПОЗИТОВ ПОЛИМЕР/УГЛЕРОДНЫЕ НАНОТРУБКИ
}

\author{
(C) 2018 Л. Б. Атлуханова ${ }^{1}$, Г. В. Козлов ${ }^{2}$ \\ 'Дагестанский государственный медицинский университет, пл. Ленина, 1, 367000 Махачкала, Россия \\ e-mail:bremovna77@mail.ru \\ ${ }^{2}$ Кабардино-Балкарский государственный университет им. Х. М. Бербекова, ул. Черныпевского, 173, \\ 360004 Нальчик, Россия \\ e-mail:i_dolbin@mail.ru
}

Поступила в редакцию 28.05.2018

\begin{abstract}
Аннотация. Выполненные оценки показали, что для полимерных нанокомпозитов понятия плотноупакованных и высокомодульных межфазных областей эквивалентны. Это обстоятельство делает указанные области армирующей компонентой нанокомпозитов. Плотноупакованные области структуры полимерной матрицы не дают вклад в усиление указанного класса полимерных материалов. Данный постулат подтвержден расчетом степени усиления нанокомпозитов в рамках перколяционной модели в предположении, что их армирующим элементом является суммарная доля нанонаполнителя и межфазных областей.
\end{abstract}

Ключевые слова: нанокомпозит, углеродные нанотрубки, межфазные области, перколяция, тангенс потерь, степень усиления.

DOI: https://doi.org/10.17308/kcmf.2018.20/573

\section{ВВЕДЕНИЕ}

В настоящее время хорошо известно [1, 2], что межфазные области в полимерных нанокомпозитах являются таким же армирующим элементом их структуры, как и собственно нанонаполнитель. Кроме того, со структурной точки зрения межфазные области можно рассматривать как структурно отличающиеся от объемной полимерной матрицы и, как правило, более плотноупакованные [3, 4] или кристаллизующиеся [1]. Для оценки относительной доли межфазных областей $\varphi_{\text {if }}$ могут быть использованы несколько методик, рассматривающих их с указанных выше позиций. Так, перколяционная модель усиления нанокомпозитов применяет для этой цели следующее соотношение [5]:

$$
\frac{E_{n}}{E_{m}}=1+11\left(\varphi_{n}+\varphi_{i f}\right)^{1,7},
$$

где $E_{n}$ и $E_{m}-$ модули упругости нанокомпозита и матричного полимера соответственно (отношение $E_{n} / E_{m}$ принято называть степенью усиления нанокомпозита), $\varphi_{n}$ - объемное содержание нанонаполнителя.
Из соотношения (1) непосредственно следует, что перколяционная модель усиления рассматривает межфазные области как армирующий элемент структуры на равных условиях с нанонаполнителем. При трактовке указанных областей как плотноупакованных для оценки их толщины $l_{i f}$ можно использовать следующее уравнение [3]:

$$
\operatorname{tg} \delta_{n}=\operatorname{tg} \delta_{m}\left[1-\left(1+\frac{l_{i f}}{r_{\mathrm{CNT}}}\right)^{2} \phi_{n}\right],
$$

где $\operatorname{tg} \delta_{n}$ и $\operatorname{tg} \delta_{m}$ - тангенс угла механических потерь нанокомпозита и матричного полимера соответственно, $r_{\mathrm{CNT}}-$ радиус углеродной нанотрубки.

Целью настоящей работы является проверка идентичности обоих рассматриваемых методов оценки характеристик межфазных областей и выяснение их влияния на свойства нанокомпозитов полимер/углеродные нанотрубки.

\section{ЭКСПЕРИМЕНТАЛЬНАЯ ЧАСТЬ}

В качестве нанонаполнителя использованы многослойные углеродные нанотрубки (МУНТ), имеющие внешний диаметр 15-20 нм, внутренний 
диаметр 5-10 нм и длину 0.5-20 мкм. Эти МУНТ были функционализированы нонборненом для повышения уровня межфазной адгезии полимерная матрица-нанонаполнитель. Содержание МУНТ составляло 0.05-0.40 масс. \%. В качестве полимерной матрицы применялся полидициклопентандиен (ПДЦПД) в эластомерном состоянии [3].

Для получения нанокомпозитов функционализированные МУНТ диспергировались в водном растворе ПДЦПД и подвергались обработке ультразвуком для улучшения диспергирования нанонаполнителя. Затем эта смесь перемешивалась с катализатором (дихлор-(3-метил-2-бутенилидин) бис-(три-циклофентил) фосфином рутения) до получения однородного раствора и сшивалась в течение 2 часов при 343 К и 1.5 часа при 443 К [3].

Механические испытания на одноосное растяжение выполнены на универсальной испытательной машине Instron 5569 согласно ASTM D638 (образцы типа V) при температуре 450 К и скорости ползуна 1 мм/мин. Каждый результат был получен как усреднение данных четырех испытаний [3].

Динамический механический анализ (ДМА) выполнен с использованием прибора TA Instruments модели Q800 DMA. Образцы испытаны на растяжение с частотой 1 Гц в интервале температур 303-583 К при скорости нагрева 3 К/мин. Образцы имели размеры $35 \times 5 \times 1$ мм [3].

\section{РЕЗУЛЬТАТЫ И ИХ ОБСУЖДЕНИЕ}

Для оценки необходимого для расчета согласно уравнениям (1) и (2) параметра $\varphi_{n}$ использована следующая формула [5]:

$$
\varphi_{n}=\frac{W_{n}}{\rho_{n}},
$$

где $W_{n}$ - массовое содержание углеродных нанотрубок, $\rho_{n}-$ их плотность, определяемая следующим образом [5]:

$$
\rho_{n}=188\left(D_{\mathrm{CNT}}-d_{\mathrm{CNT}}\right)^{1 / 3} \kappa \Gamma / \mathrm{M}^{3},
$$

где $D_{\mathrm{CNT}}$ и $d_{\mathrm{CNT}}-$ наружный и внутренний диаметр углеродной нанотрубки соответственно.

Уравнение (2) предполагает определение параметра $l_{i f}$, для оценки объемного содержания межфазных областей $\varphi_{i f}$ (в дальнейшем обозначаемого как $\left.\varphi_{i f_{2}}\right)$ авторы [1] предложили следующую формулу:

$$
\varphi_{i f 2}=\left(\frac{l_{i f}^{2}+2 r_{\mathrm{CNT}} l_{i f}}{r_{\mathrm{CNT}}^{2}}\right) \varphi_{n}+\varphi_{0},
$$

где $\varphi_{0}$ - относительное содержание упорядоченных или кристаллизующихся областей структуры на- нокомпозита, не связанных с углеродными нанотрубками.

Для определения параметра $\varphi_{0}$ сначала было определено относительное содержание межфазных областей $\varphi_{i f}$ согласно перколяционной модели (уравнение (1)), которое в дальнейшем обозначается как $\varphi_{i f 1}$, а затем построена зависимость $\varphi_{i f 1}\left(\varphi_{n}\right)$, показанная на рис. 1. Как следует из графика этого рисунка, величина $\varphi_{0}=0.070$ для малых $\varphi_{n}$, а затем увеличивается до 0.124 при $\varphi_{n}=0,010$.

На рис. 2 приведено сравнение относительных долей межфазных областей $\varphi_{i f 1}$ и $\varphi_{i f 2}$, рассчитанных согласно уравнениям (1) и (5) соответственно. Как следует из приведенных на этом рисунке данных, получено хорошее соответствие результатов, оцененных согласно двум описанным выше методикам (среднее расхождение $\varphi_{i f 1}$ и $\varphi_{i f 2}$ не превышает $10 \%$ ). Это обстоятельство указывает на эквивалентность понятий плотноупакованные (кристаллизующиеся) и высокомодульные межфазные области, которые являются армирующим элементом структуры нанокомпозита наряду с нанонаполнителем. Для проверки этого постулата был рассчитан модуль упругости нанокомпозитов ПДЦПД/МУНТ $E_{n}$ согласно уравнению (1) при $E_{m}=16.3$ МПа [3], где величина $\varphi_{i f}\left(\varphi_{i f 2}\right)$ была оценена согласно урав-

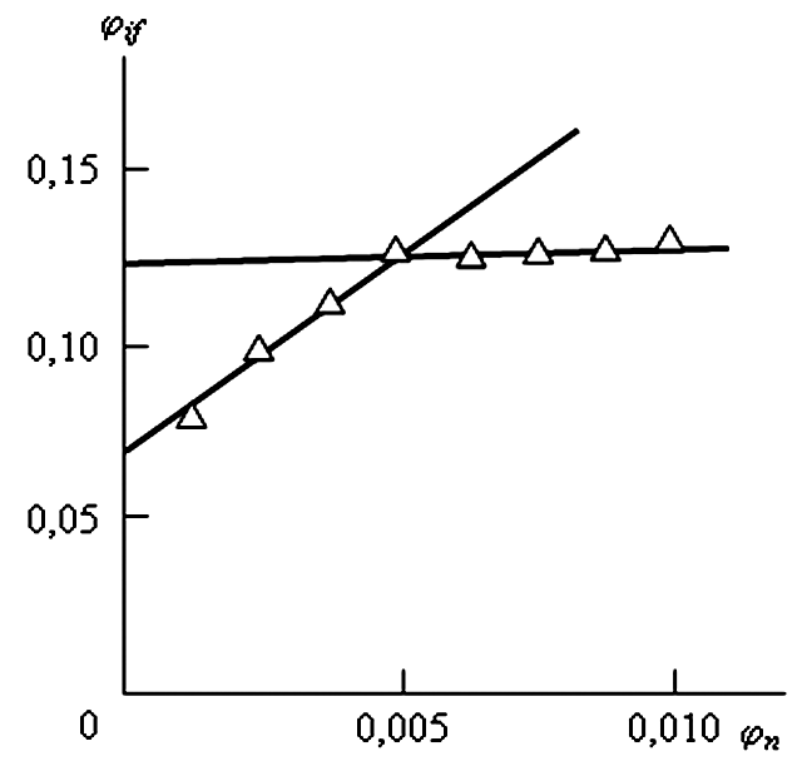

Рис. 1. Зависимость относительного содержания межфазных областей $\varphi_{i f 1}$ (уравнение (1)) от объемного содержания нанонаполнителя $\varphi_{n}$ для нанокомпозитов ПДЦПД/МУНТ

[Fig. 1. The dependence of the relative fraction of interfacial regions $\varphi_{i f 1}$ (the equation (1)) on volume contents of nanofiller $\varphi_{n}$ for nanocomposites PDCPD/MWNT] 


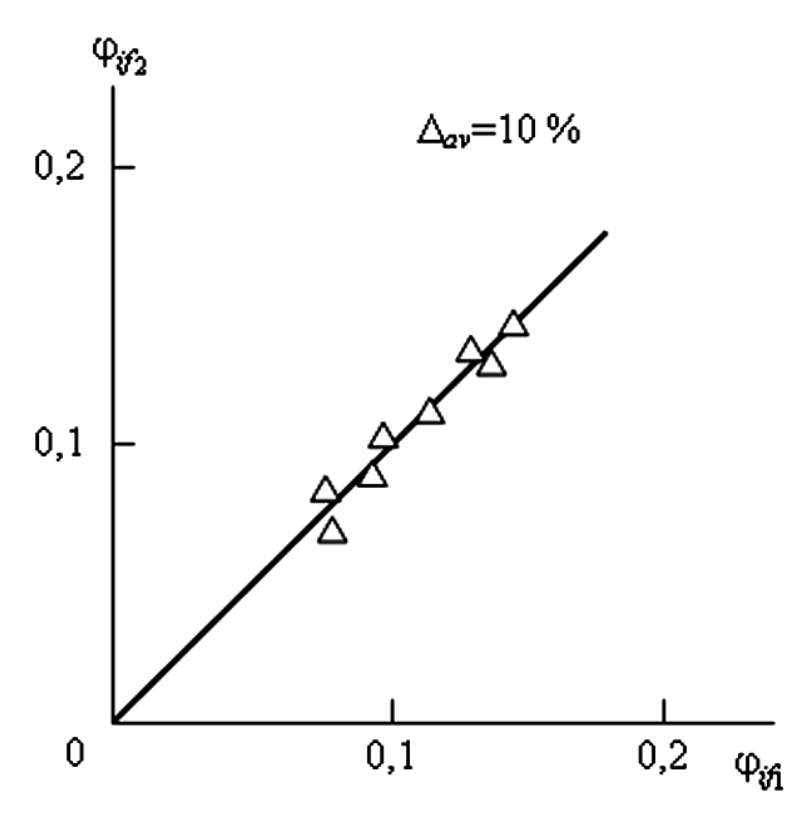

Рис. 2. Сравнение рассчитанного согласно уравнениям (1) $\varphi_{i f 1}$ и (5) $\varphi_{i f 2}$ относительного содержания межфазных областей для нанокомпозитов ПДЦПД/МУНТ

[Fig. 2. The comparison of calculated according to the equations (1) $\varphi_{i f 1}$ and (5) $\varphi_{i f 2}$ relative contents of interfacial regions for nanocomposites PDCPD/MWNT]

нению (5). Как можно видеть, получено хорошее соответствие теории и эксперимента (их среднее расхождение составляет $\sim 5 \%$ ), что подтверждает сделанный выше вывод относительно эквивалентности определения межфазных областей в рассматриваемых нанокомпозитах.

И в заключение рассмотрим два методологических аспекта. Увеличение параметра $\varphi_{0}$ по мере повышения $\varphi_{n}$ (рис. 1) приводит к снижению $\varphi_{i f 2}$, т. е. уменьшению содержания армирующей компоненты, что служит причиной снижения модуля упругости нанокомпозитов $E_{n}$. На рис. 3 штриховой линией показана гипотетическая зависимость $E_{n}\left(\varphi_{n}\right)$, полученная при условии $\varphi_{0}=\mathrm{const}=0.070$. Как можно видеть, выполнение указанного условия привело бы к повышению модуля упругости нанокомпозита ПДЦПД/МУНТ с $\varphi_{n}=0.010$ примерно на 20 \%. Таким образом, увеличение относительной доли упорядоченных или кристаллизующихся областей, не связанных с покрытием углеродных нанотрубок, снижает модуль упругости нанокомпозитов. Их полное отсутствие могло бы привести к увеличению $E_{n}$ более чем на $60 \%$ - 35.3 МПа вместо полученной эксперименталь-

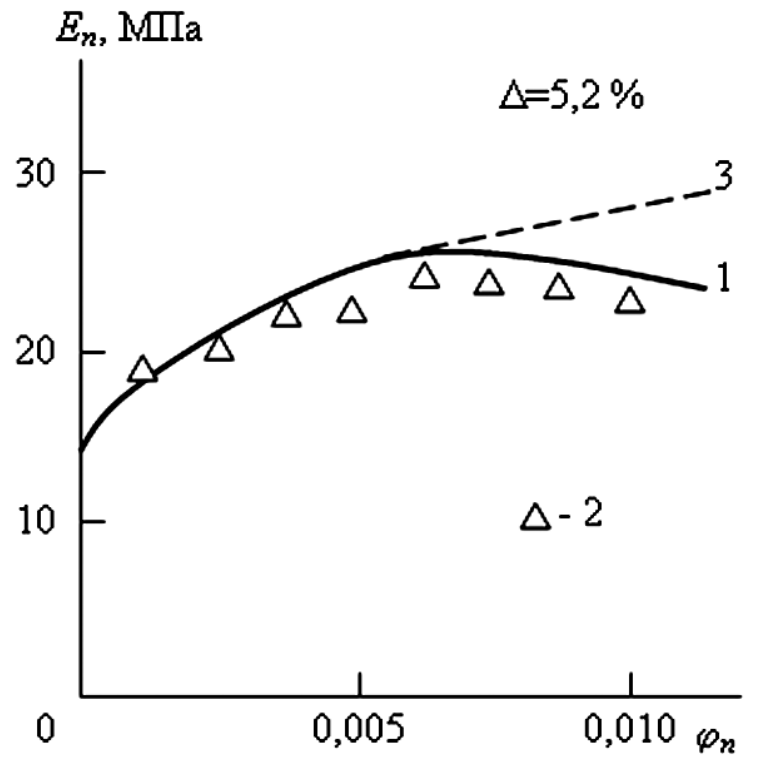

Рис. 3. Зависимости модуля упругости $E_{n}$ от объемного содержания нанонаполнителя $\varphi_{n}$, полученная экспериментально (1) и рассчитанные согласно уравнению (1) при $\varphi_{i f}=\varphi_{i f 2}(2)$ и $\varphi_{i f}=\varphi_{i f}=\varphi_{i f 1}$ при $\varphi_{0}=\mathrm{const}=0.070$ (3) для нанокомпозитов ПДЦПД/МУНТ

[Fig. 3. The dependence of modulus of elasticity $E_{n}$ on volume contents of nanofiller $\varphi_{n}$, obtained experimentally (1) and calculated according to the equation (1) at $\varphi_{\text {if }}=\varphi_{\text {if } 2}$ (2) and $\varphi_{i f}=\varphi_{i f 1}$ at $\varphi_{0}=$ const $=0.070$ (3) for nanocomposites PDCPD/MWNT]

но величины 22.4 МПа при содержании МУНТ 0.4 масс. \% [3].

Второй из указанных выше аспектов касается оценки величины $\varphi_{n}$. Наиболее часто для этой цели используется уравнение (3), особенно для малых (порядка 1 масс. \%) значений $W_{n}$, где в качестве $\rho_{n}$ применяются величины порядка 2000 кг $/ \mathrm{M}^{3}$ [6]. Однако выполненные согласно изложенной выше методике оценки при использовании в уравнении (3) величины $\rho_{n} \approx 2000$ кг $/ \mathrm{M}^{3}$ дали в конечном итоге значения $E_{n}=20.5-45.0$ МПа для рассматриваемых нанокомпозитов ПДЦПД/МУНТ, что существенно выше полученных экспериментально величин $E_{n}=18.8-22.4$ МПа [3]. Это означает, что часто ис-

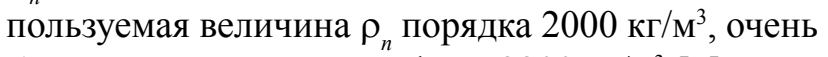

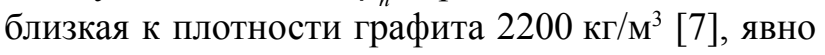
завышена, что естественным образом приводит к заниженным значениям $\varphi_{n}$. Отметим, что только учет внутренних полостей углеродных нанотрубок определяет повышение $\varphi_{n}$ в 1.5 раза [7].

\section{ЗАКЛЮЧЕНИЕ}

Таким образом, результаты настоящей работы продемонстрировали, что понятия плотноупа- 
кованных (кристаллизующихся) и высокомодульных межфазных областей эквивалентны, что дает возможность трактовать указанные области как армирующий элемент структуры нанокомпозита наряду с нанонаполнителем. Оценка относительной доли межфазных областей в рамках этих двух представлений показала их хорошее количественное соответствие. В то же время плотноупакованные области, не имеющие отношения к межфазным областям, не вносят вклад в усиление нанокомпозитов. Методы оценки свойств нанокомпозитов в значительной степени зависят от допущений, принимаемых при расчете объемного содержания нанонаполнителя.

\section{СПИСОК ЛИТЕРАТУРЫ}

1. Coleman J. N., Cadek M., Ryan K. P., Fonseca A., Nady J. B., Blau W. J., Ferreira M. S. // Polymer, 2006, vol. 47, № 23. pp. 8556-8561.

2. Иржак Т. Ф., Иржак В. И. // Высокомолекулярные соединения A, 2017, т. 59, № 6, с. 485-522. DOI: 10.7868/ S2308112017060049

3. Jeong W., Kessler M. R. // Chem. Mater., 2008. vol. 20 , № 22 , pp. 7060-7068.

4. Козлов Г. В., Долбин И. В. // Известия высших учебных заведений. Физика, 2017, т. 60, № 6, с. 72-76.

5. Микитаев А. К., Козлов Г. В., Заиков Г. Е. Полимерные нанокомпозиты: многообразие структурных форм и приложений. М.: Наука, 2009, 278 с.

6. Koerner H., Liu W., Alexander M., Mirau P., Dowty H., Vaia R. A. // Polymer, 2005, vol. 46, № 12, pp. 4405-4420.

7. Schaefer D. W., Zhao J., Dowty H., Alexander M., Orler E. B. // Soft Mater., 2008, vol. 4, № 10, pp. 20712079.

\title{
A COMPARATIVE ANALYSIS OF THE CONTENTS OF INTERFACIAL REGIONS AND THEIR INFLUENCE ON THE PROPERTIES OF NANOCOMPOSITES POLYMER/CARBON NANOTUBES
}

\author{
(C) 2018 L. B. Atlukhanova ${ }^{1}$, G. V. Kozlov ${ }^{2}$ \\ ${ }^{1}$ Dagestan State Medical University, 1 Lenina pl., 367000 Makhachkala, Russia \\ e-mail:bremovna77@mail.ru \\ ${ }^{2}$ Kh. M. Berbekov Kabardino-Balkarian State University, 173 Chernyshevski str., 360004 Nal'chik, Russia \\ e-mail:i_dolbin@mail.ru
}

Received 28.05.2018

\begin{abstract}
Nowadays, the significance of interfacial regions in the formation of polymer nanocomposites properties is internationally recognized. Structurally, these regions can be considered from two points of view: as regions, whose packing density is higher than the corresponding characteristic for bulk polymer matrix, and as a reinforcing element of nanocomposite structure, whose elasticity modulus is considerably higher than the modulus of the indicated polymeric matrix. This paper presents the results of the comparison of relative fractions of interfacial regions, obtained within the framework of the two above mentioned approaches. To determine the indicated parameter, the angular tangent of mechanical loss is used in the the first approach whereas the second approach uses the reinforcement degree of nanocompositespolydicyclopentadiene/carbon nanotubes with an elastomeric polymeric matrix. Both methods of calculation of relative fraction of interfacial regions demonstrated good correspondence. This circumstance indicates the equivalence of notions of densely-packed (crystallizing) and high-modulus (reinforcing) interfacial regions. This postulate was confirmed by the calculation of the reinforcement degree of the considered nanocomposites within the framework of percolative model of reinforcement, where the reinforcing element of nanocomposites structure is the total relative fraction of nanofiller (carbon nanotubes) and interfacial regions. The reinforcement degree calculated by the indicated method shows good correspondence with the experimental results. In conclusion, let us consider two important methodological aspects. Firstly, the ordered (or crystallizing) regions of polymeric matrix, not included in interfacial regions, do not serve as a reinforcing element of the nanocomposite structure. This means that the determination of the relative fraction of interfacial regions within the framework of the percolative model of reinforcement does not take into consideration the level of local order of the bulk polymeric matrix. Secondly, precise
\end{abstract}


calculation of carbon nanotube density is required to estimate their volumetric content. Using traditional values of the parameter for this purpose leads to artificially lowered values of nanocomposites characteristics.

Keywords: nanocomposite, carbon nanotubes, interfacial regions, percolation loss tangent, reinforcement degree.

DOI: https://doi.org/10.17308/kcmf.2018.20/573

\section{REFERENCES}

1. Coleman J. N., Cadek M., Ryan K. P., Fonseca A., Nady J. B., Blau W. J., Ferreira M. S. Polymer, 2006, vol. 47, no. 23. pp. 8556-8561. DOI: $10 / 1016 /$ j.polymer.2006.10.014

2. Irzhak T. F., Irzhak V. I. Vysokomolekulyarnye Soedineniya A [Polymer Science, Series A], 2017, vol. 59, no. 6, pp. 791-825. DOI: 10.1134/s0965545x17060049

3. Jeong W., Kessler M. R. Chem. Mater., 2008. vol. 20, no. 22, pp. 7060-7068. DOI: $10.1021 / \mathrm{cm} 8020947$.

4. Kozlov G. V., Dolbin I. V. Izvestiya Vysshikh uchebnykh zavedenii. Fizika [Russian Physics Journal], 2017, vol. 60 , no. 6 , pp. 1001-1006. DOI: 10.1007/s11182-017$1169-5$
5. Mikitaev A. K., Kozlov G. V., Zaikov G. E. Polimernye nanokompozity: mnogoobrazie strukturnykh form $i$ prilozhenii [Polymer Nanocomposites: a Variety of Structural Forms and Applications.]. Moscow, Nauka Publ., 2009, 278 p. (in Russ.)

6. Koerner H., Liu W., Alexander M., Mirau P., Dowty H., Vaia R. A. Polymer, 2005, vol. 46, no. 12, p. 44054420. DOI: $10.1016 /$ j.polymer.2005.02.025

7. Schaefer D. W., Zhao J., Dowty H., Alexander M., Orler E. B. Soft Mater., 2008, vol. 4, no. 10, p. 2071-2079. DOI: $10.1039 / \mathrm{b} 805314 \mathrm{f}$
Атлуханова Луиза Бремовна - к. п. н., доцент кафедры биофизики, информатики и медаппаратуры, Дагестанский государственный медицинский университет; тел.: +7 (963) 4020871, e-mail: bremovna77@mail.ru

Козлов Георгий Владимирович - с. н. с., Кабардино-Балкарский государственный университет им. X. М. Бербекова; тел.: +7 (8662) 424144, e-mail: i_ dolbin@mail.ru
Luiza B. Atlukhanova - Cand. Sci. (Ped.), Associate Professor of the Department of Biophysics, Informatics and Medical Devices, Dagestan State Medical University; tel.: +7 (963) 4020871; e-mail: bremovna77@mail.ru

Georgii V. Kozlov - Management of Research and Innovation Activities, Kh. M. Berbekov Kabardino-Balkarian State University; tel.: +7 (8662) 424144, e-mail: i_dolbin@mail.ru 